\title{
A rare case of paraganglioma
}

\begin{abstract}
Tumors of endocrine organs often present with a functional extremism pertaining to original functions of the gland. None can be so characteristic and at the same time fatal enough like a pheochromocytoma. Here we take the opportunity to present a rare case of a paraganglioma which presented in a much subtle form.
\end{abstract}

Keywords: pheochromocytoma, extra adrenal, paraganglioma, neuro endocrine tumors, organs, patients, prazocin
Volume 4 Issue 2 - 2017

\section{Kalyanasundarabharathi Chidambaram \\ Madras medical college, India}

Correspondence: Kalyanasundarabharathi Chidambaram, Madras medical college, 666a, mudukku street, perunagat, kanchipuram district, Tamilnadu, India, Tel 994472548I, Email kalsbhari@gmail.com

\section{Case report}

A 45 year old male was admitted to the surgical emergency ward with complaints of abdominal pain radiating to the back for the past one week, he had giddiness associated with sudden postural changes, sudden paroxysm of sweating and palpitation. No headache or vomiting. He had no co morbidities, no surgeries in the past. On examination, he was not anemic, no pedal edema, pulse rate was normal and severe hypertension $(180 / 100 \mathrm{~mm} \mathrm{Hg}$ in the left arm sitting), cardio respiratory system clinically normal, no masses in the abdomen, no swelling in the neck. Patient had fluctuating hypertension (110-190), put on anti-hypertensive. Patient had two episodes of sudden giddiness, sweating and hot flushing of face during hospital stay.

\section{Investigations}

Complete hemogram, liver and renal function tests were within normal limits, normal electrocardiograph and normal chest radiograph. His abdominal x-ray and ultrasound examinations were with no abnormality detected. Urinary 24 hours vanillyl mandelic acid was elevated $(18 \mathrm{mg} /$ day against $2-8 \mathrm{mg}$ /day). His chest CT was normal. MRI abdomen and pelvis showed a well-defined, heterogeneously enhancing retroperitoneal mass lesion seen beyond the aortic bifurcation at the level of the right common iliac artery abutting the common iliac artery and the IVC with areas of flow voids. Other solid organs normal patient diagnosed extra adrenal pheochromocytoma (paraganglioma). Put on alpha blocker- prazocin $1 \mathrm{mg}$ and enalapril $5 \mathrm{mg}$. Beta blockers were withheld as the patient's pulse \& BP standardized on Alpha blockers itself. Patient was taken up for laparotomy for excision of the tumor. Trans peritoneal approach was followed.

Intra operatively there were wide BP fluctuations (from 50 to 210 $\mathrm{mm} \mathrm{Hg}$ Systolic). Direct vascular connections from right common iliac artery and venous tributaries to IVC were ligated. Tumor was removed en-bloc. Post operatively maintained the blood pressure with tapering of alpha blockers. Patient had an uneventful post-operative recovery. The histopathology of the specimen confirmed the diagnosis of Paraganglioma (Figure 1-7).

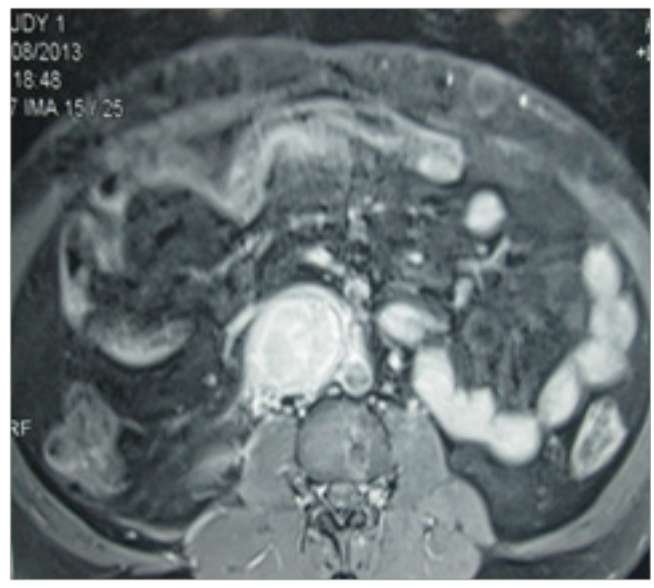

Figure I MRI abdomen axial-paraganglioma.

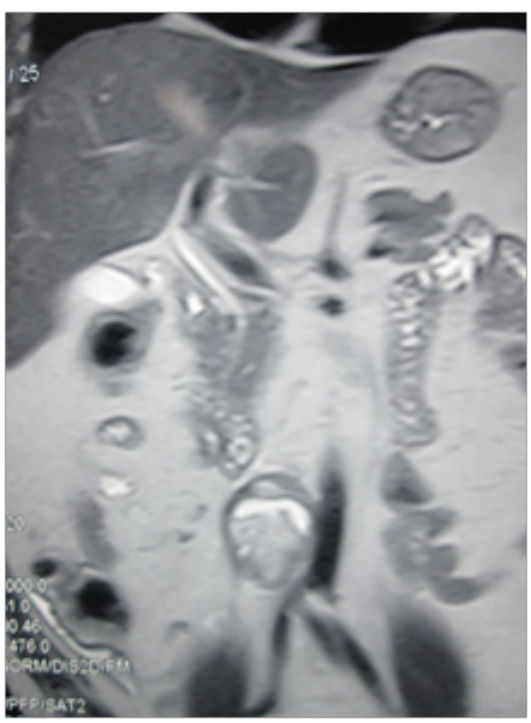

Figure 2 MRI abdomen coronal- paraganglioma near aortic bifurcation. 


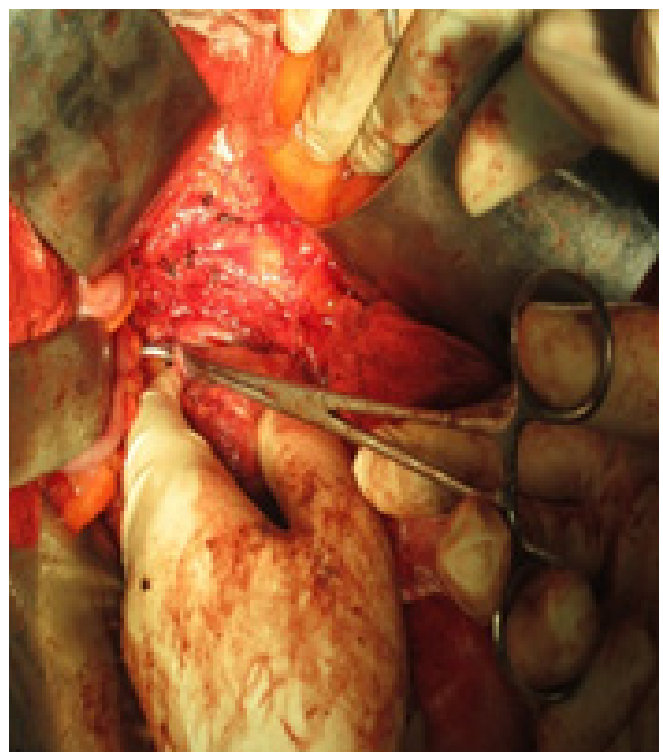

Figure 3 Lligation of feeding vessels.

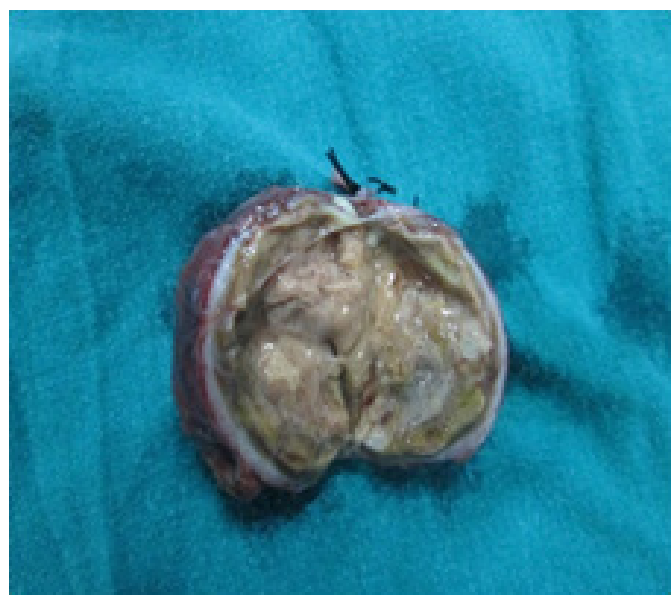

Figure 4 Specimen of the tumor.

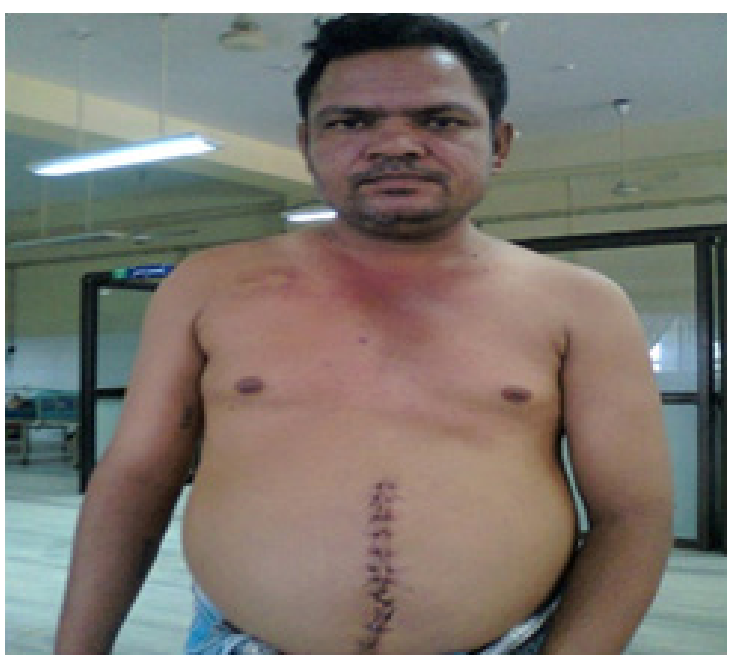

Figure 5 Patient with laparatomy wound.

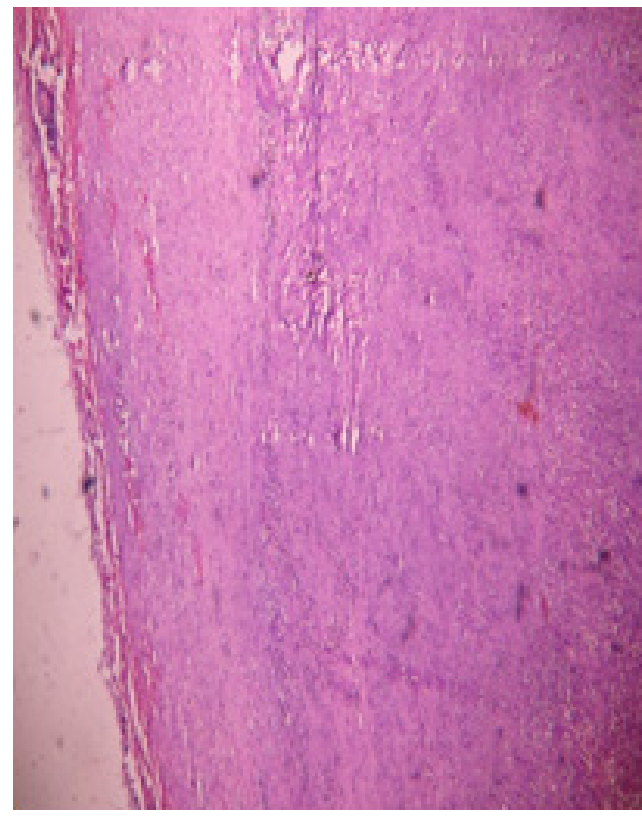

Figure 6 Histopatholgy showing well encapsulated tumor.

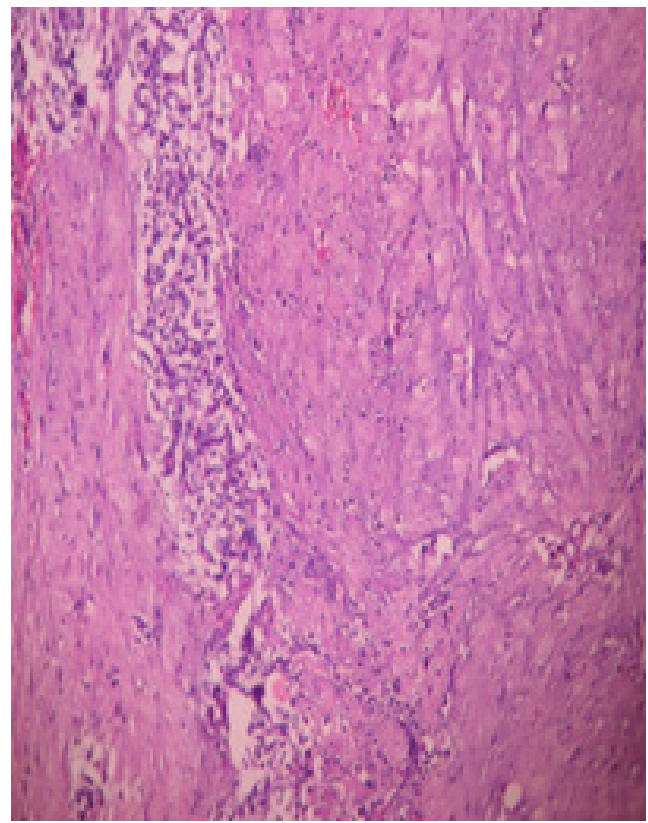

Figure 7 Histopathology - Paraganglioma.

\section{Discussion}

Phaeochromocytoma is a functioning tumor arising in the catecholamine producing chromaffin cells derived from neural crest ectodermal in origin). The first case described by Frankel in 1886 was an 18 year old presented with hypertension, sudden collapse and death and postmortem demonstrated bilateral adrenal tumors. ${ }^{1}$ This is explained by the paroxysmal release of excess catecholamines into systemic circulation. This functional extremism can also present with massive myocardial infarction and cerebrovascular events. Intraabdominal tumors constitute $98 \%$ of the phaeochromocytomas and the rest are 
found in the neck and mediastinum. Of the intraabdominal tumors more than $85 \%$ are adrenal tumors. The remaining is distributed along the sympathetic ganglia chain in the retroperitoneum. These extraadrenal tumors are called paragangliomas. Pheochromocytoma has an incidence of 2 to 8 cases per million person's annually. ${ }^{2}$ Of this pheochromocytoma arising in extra adrenal locations is less than $10 \%$ only. It is mostly discovered during autopsy/ incidental workup. 75\% died suddenly from either myocardial infarction or a cerebrovascular catastrophe. One third of the sudden deaths occurred during or immediately after unrelated minor operations.

This is an interesting case as the patient had presented with features of acute abdomen and only subtle features of such a lethal tumor with the classic triad of headache, episodic sudden perspiration and tachycardia. Hypertension is the commonest clinical sign though it contributes less than $0.5 \%$ of the newly diagnosed hypertensive's. ${ }^{2}$ It can either be sustained mimicking essential hypertension or paroxysmal, in our case it is paroxysmal elevations in blood pressure resulting in the symptoms. In the presence of classic symptoms these patients are evaluated with blood tests and urine analysis to check for elevations in the catecholamine metabolites which when elevated as in our case points to the diagnosis. ${ }^{3}$ These tumors are bilateral in $10 \%$ cases and in view of wide range of possible locations are located with appropriate imaging. Due to the low lipid content of these tumors compared to the other adrenal tumors they may be easily distinguished from them in imaging. ${ }^{4}$ Contrast enhanced computed tomography or MRI can be used since most of the tumors are more than $3 \mathrm{~cm}$ in size. In doubtful cases MIBG (metaiodo benzyl guanidine) scintigraphy can also be useful ${ }^{5}$ Presence of malignant phaeochromocytomas is a well-established entity and no reliable histological feature is confirmatory but only presence of metastasis confirms the tumor to be malignant. Adrenal phaeochromocytomas are less likely to be malignant than paragangliomas $(10 \%$ vs. $35 \%) .{ }^{6}$ In our case patient has benign paraganglioma confirmed by absence of metastasis in other parts of the body. After confirming the diagnosis and the location of the tumor it is imperative to undertake complete surgical resection of the tumor though special preoperative preparation has to be done adequately. These patients are in a state of contracted intravascular volume and hence adequately hydrated and put on alpha adrenergic blockers like phenoxybenzamine or prazocin and beta blockers if needed to control tachycardia or cardiac arrhythmias. ${ }^{7}$ In our patient reversible alpha adrenergic blocker prazocin is used with enalapril.
Laparascopic adrenalectomy has become the gold standard except in the presence of large tumor or malignant disease. ${ }^{8}$ We preferred to resort to open laparotomy since the tumor location was very proximal to the right iliac artery and the inferior venacava. Patients are kept on post operative follow up for prolonged period to diagnose early of recurrence which is reported in more than $15 \%$ cases even after 10 years. ${ }^{9}$

\section{Acknowledgments}

None.

\section{Conflicts of interest}

The author declares there is no conflict of interest.

\section{References}

1. Franz H Messerli, Leszek Michalewicz. Clinical and experimental pheochromocytoma. JAMA. 1997;278(1):78-79.

2. Lenders JW. Phaeochromocytoma. Lancet. 2005;366(9486):665-675.

3. Lenders JW, Pacak K, Walther MM, et al. Biochemical diagnosis of pheochromocytoma: which test is best? JAMA. 2002;287(11):14271434.

4. Motta-ramirez, Remer EM, Herts BR, et al. comparison of CT findings in symptomatic and incidentally discovered pheochromocytomas. AJR Am J Roentgenol. 2005;185(3):684-688.

5. Ilias I, Divgi C, Pacak K. Current role of MIBG in the diagnosis of pheochromocytoma and medullary thyroid cancer. Semin Nucl Med. 2011;41(5):364-368

6. Scholz T, Eisenhofer G, Pacak K, et al. Clinical review: Current treatment of malignant pheochromocytoma. J Clin Endocrinol Metab. 2007;92(4):1217-1225.

7. Pacak K. preoperative management of the pheochromocytoma patient. $J$ Clin Endocrinol Metab. 2007;92(11):4069-4079.

8. Pacak K, Eisenhofer G, Ahlman H, et al. Pheochromocytoma: recommendations for clinical practice from the first international symposium. October 2005. Nat Clin Pract Endocrinol Metab. 2007;3(2):92-102.

9. Khorram-Manesh, Ahlman H, Nilsson O, et al. long term outcome of a large series of patients surgically treated for pheochromocytoma. J Inten Med. 2005;258(1):55-56. 